\title{
Yang-Mills theory à la string
}

\author{
Riccardo Capovilla ${ }^{(1)}$ and Jemal Guven ${ }^{(2)}$
}

\author{
(1) Departamento de Física \\ Centro de Investigación y de Estudios Avanzados \\ Apdo Postal 14-740, 07000 México, D. F., MEXICO \\ (2) Instituto de Ciencias Nucleares \\ Universidad Nacional Autónoma de México \\ Apdo. Postal 70-543, 04510 México, D.F,, MEXICO
}

\begin{abstract}
A surface of codimension higher than one embedded in an ambient space possesses a connection associated with the rotational freedom of its normal vector fields. We examine the Yang-Mills functional associated with this connection. The theory it defines differs from Yang-Mills theory in that it is a theory of surfaces. We focus, in particular, on the Euler-Lagrange equations describing this surface, introducing a framework which throws light on their relationship to the Yang-Mills equations.
\end{abstract}

November 5, 2018

PACS: 04.60.Ds

Dedicated to Octavio Obregón, on the occasion of his 60th birthday.

Consider a surface of codimension $N$ embedded in an ambient space. There are then $N$ normal vector fields. Let us suppose that $N$ is two or higher. The normal vectors are then defined only up to a rotation, and a sign. There is a natural $O(N)$ connection on the surface associated with this freedom known as the normal connection, or extrinsic twist. This connection possesses a curvature. While the role it plays is very different from that played by the extrinsic curvature of the surface, the normal curvature is not independent of the latter: the Ricci integrability conditions determine it completely in terms of a quadratic in the extrinsic curvature [1].

There are various interesting local geometrically invariant functionals one can construct out of the normal curvature. Several of these characterize geometrical features peculiar to particular dimensions: one such invariant, characterizing two-dimensional surfaces embedded in 
four-dimensions, is the integral of the normal curvature itself; this invariant was introduced by Polyakov in the context of a stringy description of QCD [2]. For arbitrary surface dimensions, there is a natural invariant quadratic in the normal curvature: the $O(N)$ Yang-Mills functional. Appearances are deceptive however; this is not a genuine Yang-Mills theory. The dynamical variables are the embedding functions of the surface, not the connection itself. More appropriately, one should consider the theory it defines as an induced Yang-Mills theory. This is the functional we will focus on in this note. In particular, we would like to clarify the relationship between the two theories. This situation is analogous to that for Regge and Teitelboim's model for gravity in terms of an Einstein-Hilbert action, where the embedding functions, rather than the metric, appear as the dynamical variables [3]. To spell out the parallel, we have adapted the title of their paper - Gravity à la string - to its Yang-Mills counterpart.

In particular, if the surface is four-dimensional this functional is also conformally invariant. It is one of the few low order conformal invariants of the surface geometry. Perhaps the best known among these invariants is quadratic in the Weyl tensor, which depends only on the intrinsic geometry[4]. There are also polynomial invariants - apparently not so well-known to physicists - associated with the extrinsic geometry of the surface [5]. The simplest invariants of this kind are constructed using the traceless part of the extrinsic curvature. There are two independent quartics of this form [6]. Indeed, using the Gauss-Codazzi equations for the surface, it is possible to express the Weyl invariant as a linear combination of the two. In addition, a third invariant can be associated with a non-trivial competition between a quartic in extrinsic curvature and a quadratic in gradients, neither of which alone is conformally invariant [7].

Consider a $d$-dimensional surface $\Sigma$ embedded in $R^{d+N}$, where $N \geq 2$, described parametrically as follows:

$$
x=X\left(\xi^{a}\right) .
$$

Here $x=\left(x^{1}, \ldots, x^{d+N}\right)$ are local coordinates for the ambient space, $\xi^{a}, a=1, \ldots, d$ are $d$ arbitrary coordinates on the surface, and $X=\left(X^{1}, \ldots, X^{d+N}\right)$ denote the embedding functions. The only geometrically significant derivatives of $X$ are those encoded in the metric tensor $g_{a b}=e_{a} \cdot e_{b}$ and the extrinsic curvature tensor $K_{a b}{ }^{I}=e_{a} \cdot \partial_{b} n^{I}=K_{b a}{ }^{I}$, where $e_{a}=\partial_{a} X$ are the $d$ coordinate tangent vectors, and $n^{I}$ are any $N$ mutually orthogonal normal vectors $(I=1, \cdots, N)$. The Gauss-Weingarten equations describing how this surface gets embedded in the ambient space are given by $\left(\nabla_{a}\right.$ is the usual metric compatible covariant derivative on $\Sigma)$

$$
\begin{aligned}
\nabla_{a} e_{b} & =-K_{a b}^{I} n_{I}, \\
\tilde{\nabla}_{a} n^{I} & =K_{a b}{ }^{I} g^{b c} e_{c} .
\end{aligned}
$$

We have introduced the $O(N)$ covariant derivative on the surface, $\tilde{\nabla}_{a}$, associated with its invariance under rotations of the normal vectors:

$$
\tilde{\nabla}_{a} \Phi^{I}=\partial_{a} \Phi^{I}+A_{a}{ }^{I}{ }_{J} \Phi^{J},
$$


with $\Phi^{I}$ an arbitrary normal scalar, and where the normal connection is given by

$$
A_{a}^{I J}=n^{I} \cdot \partial_{a} n^{J}=-A_{a}{ }^{J I},
$$

of course for $N=1$ it vanishes identically. We denote by $F_{a b}{ }^{I J}$ the normal curvature associated with the normal connection $A_{a}^{I J}:\left[\tilde{\nabla}_{a}, \tilde{\nabla}_{b}\right] \Phi^{I}=F_{a b}{ }^{I J} \Phi_{J}$. Explicitly:

$$
F_{a b}^{I J}=\partial_{a} A_{b}^{I J}+A_{a}^{I K} A_{b K}{ }^{J}-(a \leftrightarrow b) .
$$

There are integrability conditions associated with the Gauss-Weingarten equations. Besides the well-known Gauss-Codazzi-Mainardi integrability conditions, when the number of extra dimensions is two or higher, one has the Ricci identities [1]:

$$
F_{a b}^{I J}=K_{a c}^{I} K_{b}^{c}{ }^{J}-(I \leftrightarrow J) .
$$

Thus the normal curvature associated with $A_{a}{ }^{I J}$ is completely determined by the induced metric $g_{a b}$ and the extrinsic curvature $K_{a b} I$.

With the normal curvature, we can construct the geometric functional ( $d V$ is the surface volume element constructed with $g_{a b}$ )

$$
I_{0}[X]=\frac{1}{4} \int d V F_{I J}^{a b} F_{a b}^{I J} .
$$

Superficially, it resembles an $O(N)$ Yang-Mills theory on the surface. It differs in the important respect that $I_{0}$ is a functional of the embedding functions $X$ and not of the connection $A_{a}{ }^{I J}$. One important consequence of this fact is that, while this functional is of first order in derivatives of the connection $A_{a}{ }^{I J}$, it is of second order in derivatives of the embedding functions $X$; this can be seen by looking at the Ricci identities (7): $F^{2} \sim K^{4}$, it is quartic in powers of the extrinsic curvature. We remark that, if $d=4$, the action $I_{0}$ is conformally invariant.

It is instructive to examine how the difference in the choice of the field variables manifests itself at the level of the Euler-Lagrange equations. We will compare the Euler-Lagrange derivative $\mathcal{E}$, determining the response of the functional $I_{0}$ to a surface deformation, $X \rightarrow X+\delta X$,

$$
\delta_{X} I_{0}=\int d V \mathcal{E} \cdot \delta X
$$

with the Euler-Lagrange derivative $\mathcal{E}^{a}{ }_{I J}$ corresponding to a variation in the connection $A_{a}{ }^{I J} \rightarrow$ $A_{a}{ }^{I J}+\delta A_{a}^{I J}$,

$$
\delta_{A} I_{0}=\int d V \mathcal{E}^{a}{ }_{I J} \delta A_{a}^{I J} .
$$

The first obvious thing to note is that the two differ in the number of independent variations: $d+N$ in the former versus $d \times N(N-1) / 2$ in the latter. For the latter, using the expression $\delta_{A} F_{a b}^{I J}=2 \nabla_{[a} \delta A_{b]}^{I J}$, for the variation of the normal curvature, we have the Yang-Mills equations

$$
\mathcal{E}^{a}{ }_{I J}=\nabla_{b} F_{I J}^{a b}=0
$$


In order to obtain the variation of $I_{0}$ with respect to $X$, various strategies are possible. One could vary the connection $A_{a}^{I J}$ directly, using the variational expressions obtained e.g. in Ref. [8]. This approach, however, has the disadvantage of introducing a connection deformation which plays no role at the end, but that appears annoyingly in intermediate calculation. A second approach would be to use the Ricci identities (7) to express the normal curvature in terms of the metric and the extrinsic curvature, and then vary these geometrical quantities. However, in this way the connection with Yang-Mills theory is blurred. The strategy we adopt is to introduce auxiliary variables, along the lines first suggested in Ref. [9] in a different context. We thus construct the functional

$$
\begin{array}{r}
I\left[X, e_{a}, n^{I}, A_{a}^{I J}, g_{a b}, \lambda_{a}^{I J}, \lambda^{a b}, \lambda^{I J}, \lambda^{a}{ }_{I}, \mathcal{F}^{a}\right]=I_{0}\left[A_{a}{ }^{I J}, g_{a b}\right]-\int d V\left[\lambda_{I J}^{a}\left(A_{a}^{I J}-n^{I} \cdot \nabla_{a} n^{J}\right)\right. \\
\left.-\frac{1}{2} \lambda^{a b}\left(g_{a b}-e_{a} \cdot e_{b}\right)-\frac{1}{2} \lambda_{I J}\left(n^{I} \cdot n^{J}-\delta^{I J}\right)-\lambda^{a}{ }_{I}\left(n^{I} \cdot e_{a}\right)+\mathcal{F}^{a} \cdot\left(e_{a}-\partial_{a} X\right)\right] .
\end{array}
$$

Following the approach introduced in Ref. [9], we treat the connection $A_{a}{ }^{I J}$ as variables independent of $X$. We must then introduce Lagrange multipliers to enforce the constraints that connect $A_{a}{ }^{I J}$ to the geometry. In this approach, the induced metric $g_{a b}$, as well as the basis vectors $\left\{e_{a}, n^{I}\right\}$, are also treated as independent. The innocuous apearing constraints anchoring the tangent vectors to a derivatives of the embedding functions $X$, via the Lagrange multipliers $\mathcal{F}^{a}$ will play a very important role: they identify the conserved momentum. At first sight, the introduction of a plethora of auxiliary variables appears only to complicate matters. However, as we will see, it is downhill from here: the inplementation of the constraints is straightforward. In particular, we do not need to know the variation of any of the surface geometric tensors in terms of $\delta X$. The geometrical significance of the different Lagrange multipliers will emerge automatically.

We start by considering the variation of (12) with respect to $A_{a}^{I J}$. We obtain immediately

$$
\tilde{\nabla}_{b} F_{I J}^{a b}=\lambda^{a}{ }_{I J} \text {. }
$$

Thus the Lagrange multiplier $\lambda^{a}{ }_{I J}$ is identified with the Euler-Lagrange derivative $\mathcal{E}^{a}{ }_{I J}$ introduced above (see Eq. (11)), and it acts as a source for the Yang-Mills field. It is clear that, if $\lambda_{a}^{I J} \neq 0$, the Yang-Mills equations are not satisfied.

Next, consider variations with respect to the tangent vectors $e_{a}$ :

$$
\mathcal{F}^{a}=-\lambda^{a b} e_{b}+\lambda^{a}{ }_{I} n^{I} .
$$

This is simply an expansion for $\mathcal{F}^{a}$ in terms of the basis adapted to the surface $\left\{e_{a}, n^{I}\right\}$.

The equations for the embedding functions $X$ is the statement that $\mathcal{F}^{a}$ is covariantly conserved:

$$
\nabla_{a} \mathcal{F}^{a}=0
$$

By examining the translational invariance of the functional $I$, it is clear that $\mathcal{F}^{a}$ is the conserved momentum density. Under $X \rightarrow X+a$, where $a$ is constant, we have $I \rightarrow I+\delta I$, where

$$
\delta I=-a \cdot \int d V \nabla_{a} \mathcal{F}^{a} .
$$


In particular, note that we can decompose the conservation law (15) into its tangential and normal parts by projection and using the Gauss-Weingarten equations (2), (3):

$$
\begin{aligned}
-\nabla_{a} \lambda^{a b}+\lambda^{a}{ }_{I} K_{a}{ }^{b I} & =0, \\
\nabla_{a} \lambda^{a}{ }_{I}+\lambda^{a b} K_{a b I} & =0 .
\end{aligned}
$$

The tangential projection is the Bianchi identity associated with the reparametrization invariance of the functional $I$. The second, normal, is the Euler-Lagrange derivative for the functional $I_{0}[X]$ with respect to the embedding functions $X$ (see . e.g. [10]). What remains to be done is to identify the geometric content of the components $\lambda^{a b}$ and $\lambda^{a}{ }_{I}$. For this, consider now the variation with respect to the induced metric $g_{a b}$. It identifies the tangential component of the stress tensor $\lambda^{a b}$ with the metric stress tensor for the Yang-Mills functional (8):

$$
\lambda^{a b}=T^{a b}=F^{a c I J} F^{b I J}-\frac{1}{4} g^{a b} F_{c d}^{I J} F_{I J}^{c d},
$$

where the metric stress tensor $T^{a b}$ is defined in the usual way as

$$
T^{a b}=\frac{1}{2 \sqrt{g}} \frac{\delta I_{0}}{\delta g_{a b}} .
$$

Note that $T^{a b}$ itself is not conserved. If $d=4$, it is, however, traceless, the hallmark of conformal invariance. We find that the tangential projection of the conservation law for $\mathcal{F}^{a}$ (16) now reads

$$
\nabla_{a} T^{a b}=K_{a}^{b I} \lambda^{a}{ }_{I}
$$

The extrinsic curvature of the surface and the normal component of the momentum density provide a source for the divergence of the metric stress tensor $T^{a b}$.

To determine the normal component of $\mathcal{F}^{a}, \lambda^{a}{ }_{I}$, we consider the last variation, with respect to the normal vectors $n^{I}$ :

$$
\lambda^{a}{ }_{I J} \nabla_{a} n^{J}+\nabla_{a}\left(\lambda_{I J}^{a} n^{J}\right)+\lambda_{I J} n^{J}+\lambda^{a}{ }_{I} e_{a}=0 .
$$

The tangential projection of this equation identifies the Lagrange multiplier $\lambda^{a}{ }_{I}$, or the normal component of the momentum density, for us:

$$
\lambda^{a}{ }_{I}=-2 \lambda^{b}{ }_{I J} K_{b}^{a}{ }_{b}^{J}=-2 \mathcal{E}^{b}{ }_{I J} K_{b}^{a}{ }_{b}=-2\left(\tilde{\nabla}_{c} F^{b c}{ }_{I J}\right) K_{b}^{a}{ }^{J} .
$$

The normal projection identifies the remaining Lagrange multiplier $\lambda_{I J}$ as

$$
\lambda_{I J}=2 A_{a(I}{ }^{K} \lambda_{|K| J)}^{a},
$$

together with the vanishing of the divergence of the Lagrange multiplier $\lambda_{I J}^{a}$, or equivalently, of the Yang-Mills Euler-Lagrange derivative:

$$
\tilde{\nabla}_{a} \lambda^{a}{ }_{I J}=\nabla_{a} \mathcal{E}^{a}{ }_{I J}=0 .
$$


This is the Bianchi identity associated with the $O(N)$ invariance of the Yang-Mills functional.

We conclude that the momentum density takes the form

$$
\begin{aligned}
\mathcal{F}^{a} & =-T^{a b} e_{b}-2 K^{a}{ }_{b}{ }^{J} \mathcal{E}^{b}{ }_{I J} n^{I}, \\
& =-T^{a b} e_{b}-2 K^{a}{ }_{b}{ }^{J}\left(\nabla_{c} F^{c b}{ }_{I J}\right) n^{I} .
\end{aligned}
$$

The first line is valid for any functional of the normal connection. It requires only to determine the variations of the functional with respect to the induced metric $g_{a b}$, to obtain $T^{a b}$ via Eq. (19), and with respect to the connection to obtain $\mathcal{E}^{a}{ }_{I J}$. The second line is specific to the Yang-Mills model and $T^{a b}$ is given by (18). Note that, unless the Yang-Mills equations hold, there is a non-trivial normal component of the momentum density.

It is immediate to see that the tangential projection of the divergence of $\mathcal{F}^{a}$, (16), is an identity. The vanishing of the normal projection of the divergence of $\mathcal{F}^{a}$ produces the EulerLagrange equations of the functional $I$ with respect to $X$ (see e.g. [10]):

$$
2 \tilde{\nabla}_{a}\left[K_{b}^{a}{ }^{J}\left(\nabla_{c} F_{I J}^{c b}\right)\right]-K_{a b}^{I} T^{a b}=0 .
$$

These equations are fourth order in derivatives of the embedding functions. Even if the YangMills equations are satisfied, so that the first term vanishes, one must still contend with the second term. In this case, however, the equations reduce to second order: curiously, they are reminiscent of the Euler-Lagrange equations for the Regge-Teitelboim model [3].

In conclusion, we have described a theory of embedded surfaces described by a Yang-Mills functional. In particular, we have examined the relationship between this theory and Yang-Mills theory at the level of the Euler-Lagrange equations as well as the conserved momentum. While the two theories differ, there are also intriguing connections. A detailed discussion will be presented elsewhere. 


\section{Acknowledgments}

Partial support from CONACyT grants 44974-F, 51111 as well as DGAPA PAPIIT grant IN119206-3 is acknowledged.

\section{References}

[1] M. Spivak, A Comprehensive Introduction to Differential Geometry. Vol.Four, Second Edition (Publish or Perish, 1979).

[2] A. Polyakov, Nucl. Phys. B 268406 (1986).

[3] T. Regge and C. Teitelboim, Proceedings of the Marcel Grossman Meeting, Trieste, Italy, (1975), ed. Ruffini R (North-Holland, Amsterdam, 1977) 77.

[4] R. M. Wald, General Relativity (University of Chicago Press 1984).

[5] T.J. Willmore, Total Curvature in Riemannian Geometry (Chichester: Ellis Horwood, 1982).

[6] R. Capovilla, R. Cordero, J. Guven, Mod. Phys. Lett. A 112755 (1996).

[7] J. Guven J. Phys. A: Math. and Gen. 387943 (2005).

[8] R. Capovilla and J. Guven, Phys. Rev. D 516736 (1995).

[9] J. Guven, J. Phys. A: Math and Gen. 37 L313 (2004).

[10] G. Arreaga, R. Capovilla, and J. Guven, Ann. Phys. 279126 (2000). 